\title{
Palpation zwischen Struktur und Energie
}

\author{
Klaus G. Weber, Rottenburg
}

Palpation ist eine Kunst. Unsere palpatorischen Fähigkeiten wachsen und differenzieren sich ein Leben lang. Die Erfahrung als Ausbilder zeigt, dass die palpatorische Information nicht nur eine Frage der Sensorik ist. Es gibt keinen Grund, warum nicht alle gesunden Menschen einen annähernd gleich guten Tastbefund erheben sollten.

\section{Palpation - eine Frage der Begriffsbildung}

Es ist oft der Mangel an Begriffsbildungen, der uns daran hindert, die wahrnehmbaren bzw. die wahrgenommenen Informationen zu differenzieren und bewusst zu machen. Ohne eine sprachliche, damit auch begriffliche Unterscheidung kann sich die Wahrnehmung fein differenzierter Informationen nicht weiterentwickeln.

„Information ist ein Unterschied, der einen Unterschied macht." Dieser Satz

\section{Zusammenfassung}

Qualität und Informationsgehalt palpatorischer Befunderhebung sind abhängig von der Bereitschaft, subjektive, sich aus Transsensus und nonverbaler Interaktion ergebende Informationsebenen ernst zu nehmen und zu erschließen. Aus der Erfahrung der Ortho-Bionomie lohnt es, die Wahrnehmung eigener Interaktionsreaktionen im Kontakt mit den Patienten als Informationsquelle zu schulen. Eine vom Intellekt gesteuerte Fokussierung und die Analyse der Eindrücke eröffnen neue Kausalitäten und Therapiewege. aus der systemischen Wirtschaftstheorie lässt sich auf unsere Arbeit übertragen. Die Palpationsbefundung hängt $a b$ von einer gelungenen intellektuellen Zuordnung sensorischer Wahrnehmungen.

Den Rahmen unserer Fähigkeit, Reizinformationen bewusst zuzuordnen, bilden kulturelle, psychische und weltanschauliche Faktoren.

Einige Beispiele: Bei normalem Hörvermögen ist es einem Deutschen kaum möglich, bedeutunggebende Klangfärbungen chinesischer Wörter zu hören, weil er nicht weiß, was er hören soll. Ähnliches gilt für die Palpation. Spezifische Stressund Traumawahrnehmungen unter der Palpation (s.u.) werden im Rahmen einer Gegenübertragung oder aus weltanschaulichen Gründen instinktiv ausgeblendet. Unter weltanschaulich verstehe ich hier unser individuelles Bild von der Wirklichkeit. Was aus „wissenschaftlichen Gründen" nicht plausibel ist, was nicht sein darf, wird ausgeblendet.

Der letzte Faktor ist bedeutsamer, als es viele realisieren oder zugeben möchten. Manche beschränken die Palpation auf sog. objektive Befunde. Damit glauben sie sich in „der wissenschaftlichen Gemeinde" einen Rest von Anerkennung erhalten zu können. Subjektive Wahrnehmungsanteile werden nicht registriert, negiert und dadurch einer qualitativen Interpretation entzogen. Die objektiven Befunde decken nur ein geringes Spektrum unserer palpatorischen Möglichkeiten ab. Bei Überschreiten der Grenze des objektiven Befundes tun häufig die Beteiligten so, als ob der subjektiv, interaktiv erhobene Befund etwas Objektives sei wie z.B. die objektive Palpation des Ligamentum coronarium dexter.

Qualitativ, also subjektiv erhobene, klinisch relevante Informationen als objektiven Palpationsbefund zu bezeichnen, ist zwar psychologisch verständlich, führt aber zu kaum auflösbaren Verwirrungen. Das Verdrängen dieser sehr realen Situation erschwert es jüngeren Therapeuten, ihre Palpationsfähigkeiten gezielt und zugleich selbstkritisch zu verfeinern.

Ich möchte Sie zu einem Versuch mit einem Partner einladen. Das Erleben ist erhellender als viele Worte.

\section{Summary}

\section{Palpation between Structure and Energy}

Quality and informational content of palpatory findings depend on the willingness, to take serious und to deduce subjective information levels, which result from transsensus and nonverbal interaction. The experience gained with ortho-bionomy shows that it is worth, to train the perception of one's own interaction reactions in contact with the patients as a source of information. Focusing, controlled by intellect, and the analysis of the impressions open up new causalities and ways of therapy.

\section{Résumé}

\section{Palpation entre structure et énergie} La qualité et le taux d'information d'une enquête diagnostique palpable dépendent de la disposition de prendre au sérieux les niveaux d'information subjectifs engendrés par le transsensus et l'interaction non-verbale et de les comprendre. Avec l'expérience de l'ortho-bionomie, il est profitable d'éduquer, comme source informative, la perception de ses propres réactions interactives au contact avec les patients. Une focalisation intellectuelle et l'analyse des impressions ouvrent de nouvelles causalités et voies thérapeutiques. 


\section{Exemplarische Palpationsübung}

Bei der Palpation nahe am Ellbogen sollen Sie sich achtsam durch die einzelnen Körperschichten bis zum Knochenkontakt einsinken lassen. Die Fingerendglieder liegen gelenknah flächig auf dem Arm. Der Palpationsdruck darf variieren.

Lassen Sie alle sensorischen und emotionalen Assoziationen zu. Jede Schicht, jede Qualität wird von Ihnen benannt und beschrieben. Ihr Partner soll rückmelden, ob er Ihre Eindrücke teilt, sie nachvollziehen kann oder ob er sie anders empfindet.

\section{Haut}

Ertasten Sie die Haut, schieben Sie sie leicht hin und her, spüren gegebenenfalls die feinen Härchen auf der Haut. Wie sind die Spannung, die Temperatur, die Schichtdicke, der Turgor? Ist die Haut präsent? Fällt es Ihnen leicht, sich nur die Haut vorzustellen, sich auf sie einzulassen oder drängt sich eine tiefere Schicht unwillkürlich in Ihrer Wahrnehmung vor?

\section{Unterhaut und Oberflächenfaszie}

Wie ist der Übergang von der Haut zum Unterhautgewebe, zur Oberflächenfaszie: leicht und fließend, scharf markiert, nicht zu trennen oder anders zu beschreiben? Wie wirkt die Oberflächenfaszie? Im Sei-

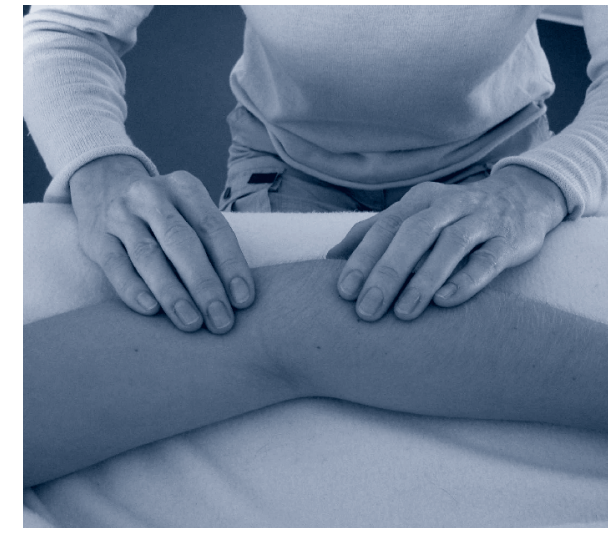

O 1 Palpation des Ellbogens.

tenvergleich zeigen sich oft große Unterschiede. Eine straffe, trennende Faszie setzt der tieferen Wahrnehmung einen Widerstand entgegen. Sie kann auch weich zur darunterliegenden Muskulatur überleiten. Empfinden Sie Stresswahrnehmungen bei erhöhtem Tonus oder bei Tonusverlust?

\section{Muskulaur}

Unter einer sehr straff und trennend wahrgenommenen Oberflächenfaszie ist der Muskel oft mehr in seinen faszialen Anteilen zu spüren als mit dem Parenchym. Es finden sich Diskrepanzen zwischen objektiver Größe und Ausprägung der Muskeln und ihrer Präsenz unter den Fingern. Manchmal entsteht sofort ein Knochenkontakt, als wäre die Muskulatur räumlich kaum vorhanden. In anderen Fällen wirkt ein Muskel plastisch, elas- tisch, teigig, homogen, vielschichtig wie ein Bündel, lebendig, erschöpft, unruhig, angespannt, wie gemeißelt und vieles andere mehr.

\section{Periost und Knochen}

Gelegentlich trifft man vor dem Knochengefühl auf einen zäh-elastischen Widerstand, das Periost. Dessen scheinbare Dicke kann in der subjektiven Präsenz sehr unterschiedlich sein. Zu den bemerkenswerten, nicht seltenen Befunden gehören der überpräsente Knochen und die erschwerte bzw. Nichtwahrnehmung des Knochens. Objektiv findet man den harten Widerstand, hat aber keinen qualitativen Kontakt mit dem Knochen. Den Patienten ist es oft unangenehm, wenn der Untersucher sich jetzt noch mehr fokussiert, sich anstrengt den Knochen wahrzunehmen. Der Palpationsdruck bleibt unverändert.

\section{Therapeutische Konsequenzen}

Subjektive Eindrücke neben dem „objektiven" Befund sind therapeutisch von großem Belang. Eine überpräsente Muskulatur und ein in den Hintergrund tretender Knochen werden anders $\mathrm{zu}$ behandeln sein als eine kaum präsente Muskulatur und eine dominierende Knochenwahrnehmung. Dahinter kann eine Überlastung stecken, eine posttraumatische Stressreaktion oder das Ausblen- 
den aus dem Körpermuster aus ähnlichen Gründen. Die Symptomatik mag sich ähneln, das Vorgehen in der Behandlung unterscheidet sich. Eine im Untersucher anklingende emotionale Resonanz, das Gefühl von inneren Zusammenhängen mit anderen Körperstrukturen sind weitere bedeutsame Informationen. Muskuläre, ossäre, fasziale, viszerale Funktionsketten machen sich unter der Palpation bemerkbar, erschließen neue Behandlungsaspekte.

\section{Fokus und Kontakt}

Als Therapeuten sprechen wir bei aller angestrebten Ganzheitlichkeit in der Arbeit meist nur Einzelaspekte der Wirklichkeit des Patienten an. Entscheidend ist die bewusste Wahl, der Fokus mit dem wir arbeiten - strukturell, funktionell-dynamisch oder energetisch. Ich bin immer erstaunt, wie sehr diese intellektuelle Entscheidung zu unterschiedlichen Kontakt- und Wirkqualitäten führt. Die Berührung ist das therapeutische Mittel, mit dem wir unsere Wahl realisieren. Nach der physikalischen Definition der Energie als jene Kraft, die eine Änderung zu bewirken vermag, ist der aktive Intellekt demnach eine echte Energieform.

Die Palpation leitet direkt über zur Therapie. Während die therapeutische Intervention selektiv definierte Ebenen und Funktionen anspricht und damit sehr fokussiert ist, sollte die Palpation möglichst offen sein für alle Eindrücke. Es geht um eine Offenheit, alle Eindrücke unter der Palpation als solche zu registrieren, und sie zunächst möglichst wenig zu bewerten und zuzuordnen. Strukturelle, mehr „objektive“ Befunde besitzen keine höhere oder geringere Wertigkeit als die mehr subjektiven energetischen Eindrücke.

\section{Strukturelles Arbeiten - Die „objektive“ Arbeit am Objekt}

Wenn wir Menschen biomechanisch wie eine unbelebte Maschine betrachten, die auf objektive Reize objektiv reagiert, so wird dieser Ansatz unser therapeutisches Vorgehen prägen. Gut messbare und quantifizierbare Energie, ob thermisch, elektrisch oder mechanisch, wirkt auf die Strukturen und Funktionen, die ihrer Wirkung ausgesetzt sind. Diese Energien sollen möglichst gleichförmig bestimmte Reaktionsmuster auslösen. Selbst die „objektiven“ Energien der physikalischen Therapie wie Druck, Vibrationen, Winkelbewegungen usw. können jedoch zugleich zum Träger wirksamer qualitativer Informationen werden.

\section{Was nicht messbar ist, existiert nicht}

Schwerer zu beschreiben und einzuschätzen sind die Qualität und spezifische Wirkung einer Therapie, die den subjektiv ausgestalteten Kontakt von Therapeut und Patient einbezieht. Hier überwiegen klinisch relevante, aber mangels besseren Verständnisses immer noch als „unspezifisch“ bezeichnete Wirkungen. Die Forschung gibt physikalischen Parametern und „zwangsläufigen“ physiologischen Reaktionen wegen ihrer leichteren Messbarkeit den Vorzug. Nur was heute messbar ist, ist heute erforschbar. Nehmen Sie die Akupunktur bei Lumbalgie. Die unwirksamere Pharmakotherapie gilt trotz ihrer geringen Wirkung gegenüber Placebo als gesichert, während die viel wirksamere Akupunktur wegen ihrer hohen unspezifischen Wirkung nicht gegen die Placeboakupunktur statistisch gesichert werden konnte.

Deshalb versucht man immer noch Techniken mit intensivem Patientenkontakt wie Massagen, Krankengymnastik, OrthoBionomie, Osteopathie, Rolfing usw., v.a. unter angeblich objektivierbaren Kriterien zu beurteilen.

\section{Wodurch wird Therapie wirksam?}

Die Qualität der Interaktion ist nach unserer Erfahrung für die Wirkung wichtiger als die gewählte Technik. In den Körpertherapien spielt eine verbale Kommunikation nicht die primäre Rolle. Zu viel verbaler Austausch kann von der Körperwahrnehmung ablenken und diese stören. Nonverbal findet bei der Untersuchung und Behandlung eine ständige Kommunikation der Beteiligten statt. Die nonverbale Kommunikationsebene, die wir in der Berührung erleben, wird meist als „energetische Ebene“ bezeichnet.

\section{Therapierelevante „Energien“}

„Energie“ wird therapeutisch weiter gefasst als in der engen physikalischphysiologischen Definition. Wie in der Physik besitzt die Energie im therapeutischen Umfeld unterschiedliche Erscheinungsformen.

\section{Energiephänomene auf physikalisch-biochemischer Grundlage}

Für Wellenmuster wie Schall, Vibration, Licht/Farbe, Temperatur und Elektrizität gilt in der dynamisch-energetischen 


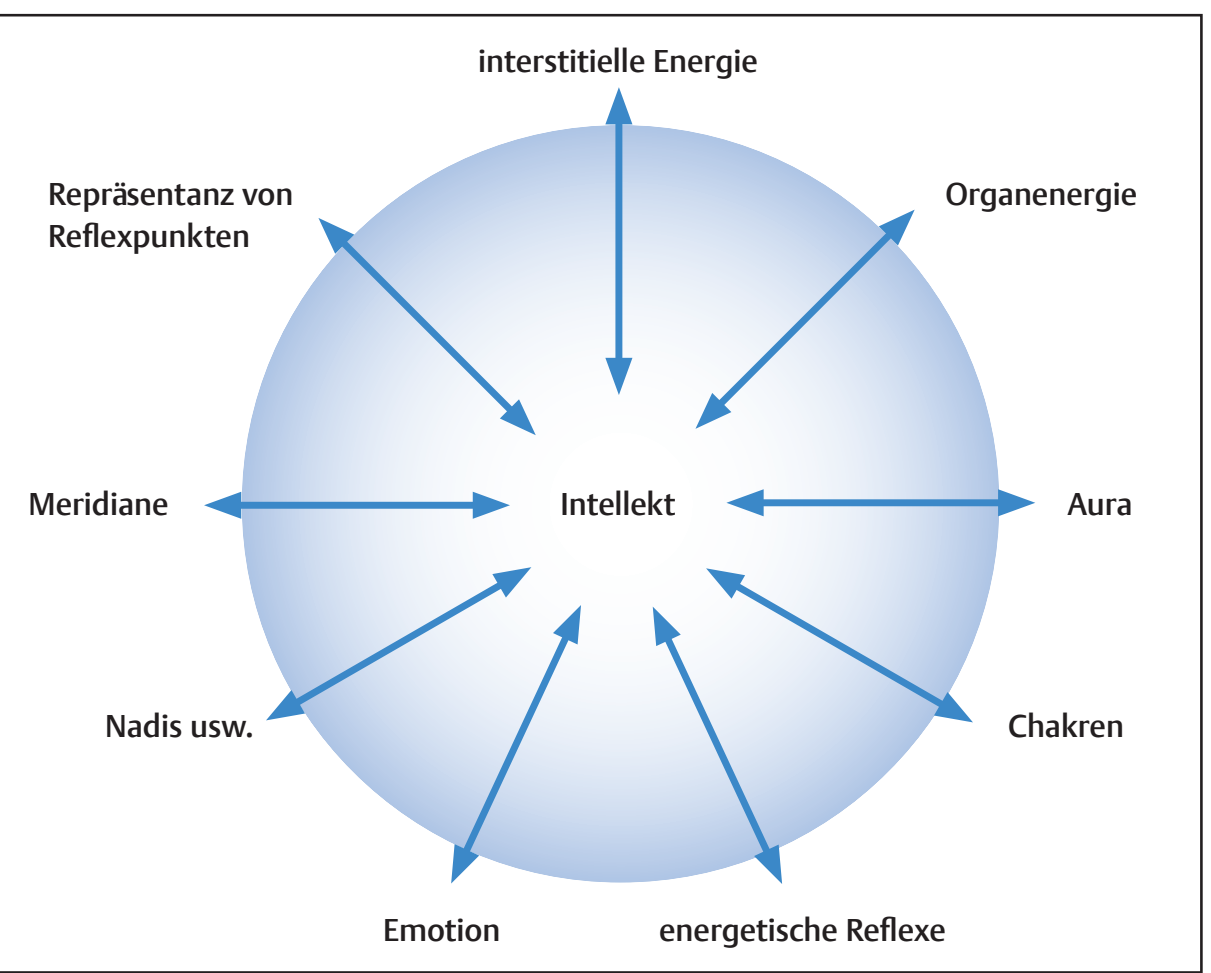

O 2 Körperliche und energetische Rhythmen.

Interaktion, dass die Wellen nicht gleichzusetzen sind mit ihrem Informationsgehalt. Neben ihrer objektivierbaren Größe und Intensität transportieren die einzelnen Energiephänomene Stimmungen, Zustandsaussagen, Informationen über Stimmigkeit und Unstimmigkeit usw.

Nehmen wir die Wärme. Durchblutungsänderungen und eine lokale Stoffwechselanregung sind übliche Phänomene. Daneben werden viele das Gefühl kennen, dass sich die Wärme bei einem Patienten als produktive Wärme, bei einem anderen als kranke Hitze vermittelt. Kühle kann sich neutral, angenehm frisch oder so anfühlen, als würde dem Behandler die Eigenwärme entzogen.

Veränderungen der Muskelspannung, Vibrationen, Pulswellen, kraniale Rhythmen treten spontan auf oder werden durch eine therapeutische Berührung induziert. Ihre subjektiven Qualitäten haben Bedeutung. Spannung kann Angst, Wut, Stress, Neugier, Lebendigkeit, Freude und anderes mehr ausdrücken. Bewegungen können schwach, zögerlich oder entspannt wirken, lebendig, gereizt oder von Entzündung getrieben und vieles mehr. Das Gleiche gilt für die Vibration, die muskuläre Entspannung usw. kulturellen Prägungen abhängig. Die Schichten in der Energie kann man mit Übung deutlich unterscheiden. Mit einer Berührung, die unseren Patienten als Person anspricht, betreten wir immer den Intimraum. Berührung verlangt Achtsamkeit, Respekt und nicht zuletzt die Zustimmung des Berührten.

\section{Transsensus - Energiewahr- nehmungen im Kontakt}

Ohne Sinnesorgan für energetische Phänomene können wir dennoch energetische Interaktionen nutzen, indem wir eigene subtile Wahrnehmungsveränderungen den energetischen Wechselwirkungen zuordnen. Kinästhetische Wahrnehmungen als Projektionen energetischer Interaktionen sind recht häufig. Jeder hat schon einmal ohne äußere Anzeichen körperlich gespürt, dass es einem Menschen im Umfeld physisch oder emotional gut oder schlecht geht. Wir nehmen das über Veränderungen des eigenen Befindens wahr.

Eine eingangs kalibrierte Selbstwahrnehmung vermittelt durch Veränderungen des eigenen Befindens klare Botschaften. Beispiele: Ein längerer oder intensiverer Kontakt ist erwünscht. Emotionen drängen in den Vordergrund. Man nähert sich der Quelle der Beschwerden...

Vorsicht ist bei der Deutung geboten. Wahrnehmungen können missverstanden werden. Kribbeln kann angenehme Spannung oder Angst bedeuten, Kühle Entspannung oder Erschöpfung, Schwingen und Kreisen Leichtigkeit oder Haltverlust. Aktives Hinterfragen der eigenen Vorurteilsbildung schafft rasch Klarheit. 


\section{Emotionale Energie}

Auf den Therapeuten übertragene Emotionen wie Angst, Mutlosigkeit, Ärger, Neid, Frustration werden im Körperkontakt spürbar, ohne darüber zu sprechen. Eine Hand auf Ihrer Schulter kann Freundlichkeit, Trauer, Trost, Unterstützung, erotisches Interesse, Dominanz und andere Gefühle vermitteln. Hier tun sich weitreichende therapeutische Möglichkeiten und Risiken auf. Berührung schafft so viel Nähe und Interaktion, dass analytische psychotherapeutische Verfahren den körperlichen Kontakt strikt ablehnen.

\section{Strukturierte Formen der Energie}

Einige traditionelle Medizinsysteme kennen eine strukturelle Organisation der menschlichen Energie (das Qi) wie die Chakren, die Nadis, das Hara sowie die Meridiane (Energiekanäle). Eine lebendige Energieverteilung und der Energiefluss halten den Organismus gesund.

Wir unterliegen vielen ordnenden Rhythmen, die auf physiologischen Parametern und auch auf rhythmische Energiebewegungen beruhen. Beide Elemente können sich voneinander trennen, sich stören, zusammenfinden, sich wechselseitig unterstützen und stärken.

Gespeicherte Angstenergie beispielsweise hemmt die körperliche Bewegung. Die Auflösung einer Traumaspannung ermög- licht im Gegenzug die freie, entspannte Bewegung. Beide Bewegungsqualitäten, die energetische und die körperliche, gleichzeitig im Kontakt wahrzunehmen, stellt eine hohe Herausforderung dar.

\section{Palpation und Berühren - Medium wechsel- seitiger energetischer Beeinflussung}

Energien wirken nicht eindirektional, fließen und interagieren nicht nur in eine Richtung. Energien zu erfahren, gehört zu unseren Orientierungs- und Selbsterhaltungsfähigkeiten. Wir sind Sender und Empfänger zugleich. Die kritische Distanz und Fokussierung auf „objektive“ Parameter ist der effektivste Mechanismus zur Ausblendung von Energieinteraktionen. Wir fassen den Patienten sachlich distanziert an, arbeiten an ihm und weniger mit ihm.

Umgekehrt kann der qualitative Kontakt unser Arbeitsmedium werden. Über das bewusste Berühren wird das Maß der wechselseitigen Interaktionen gesteuert. Man kann lernen, die Energie des Patienten wahrzunehmen, sie in Diagnostik und Therapie einzubeziehen und sich selbst wenig von ihr beeinflussen zu lassen.

\section{Wirkungen der Berührung in Palpation und Behandlung}

Die Berührung bezieht körperlich und energetisch den ganzen Organismus ein. Sie wirkt sowohl lokal als auch über körperliche und energetische Funktionsketten. Segmentalreflektorisch und über Somatotopien stimuliert sie die Selbstregulation. Sie beeinflusst das Vegetativum, die Emotionen, die Psyche. Mit der Steigerung der körperlichen Selbstwahrnehmung verbessert und differenziert sie die emotionale und intellektuelle Selbstwahrnehmung. Berühren unterstützt und verbessert auf allen Ebenen unser diagnostisches und therapeutisches Handeln im Sinne des Patienten.

Wenn wir auf unsere Grenzen und die Grenzen unserer Patienten achten, wenn wir mit beidem achtsam und freundlich umgehen, wird die körperlich-energetische Berührung für beide Seiten eine Bereicherung sein.

\section{Abbildungen:}

1 aus: Wiese M, Weber KG: Dynamische und energetische Techniken. Stuttgart: Sonntag; 2006.

Einen weiteren interessanten Beitrag zum Thema lesen Sie in: www.thieme-connect.de/ejournals/ abstract/do/doi/10.1055/s-2008-1077205 